\title{
Infinitely Many Securities and the Fundamental Theorem of Asset Pricing
}

\author{
Alejandro Balbás and Anna Downarowicz
}

\begin{abstract}
Several authors have pointed out the possible absence of martingale measures for static arbitrage free markets with an infinite number of available securities. Accordingly, the literature constructs martingale measures by generalizing the concept of arbitrage (free lunch, free lunch with bounded risk, etc.) or introducing the theory of large financial markets. This paper does not modify the definition of arbitrage and addresses the caveat by drawing on projective systems of probability measures. Thus we analyze those situations for which one can provide a projective system of $\sigma$-additive measures whose projective limit may be interpreted as a risk-neutral probability of an arbitrage free market. Hence the Fundamental Theorem of Asset Pricing is extended so that it can apply for models with infinitely many assets.
\end{abstract}

Mathematics Subject Classification (2000). 91B28, 28C15, 28A53.

Keywords. Infinitely many securities, arbitrage, martingale measure, projective system.

\section{Introduction}

The existence of pricing rules, discount factors or state prices is crucial in Mathematical Finance. It is closely related to the concepts of arbitrage and equilibrium (see, for instance, [5]). Harrison and Kreps [10] showed the link between pricing rules and martingale measures.

Since Harrison and Kreps [10] established the existence of martingale probability measures for some arbitrage-free pricing models their result has been extended in multiple directions, generating the Fundamental Theorem of Asset Pricing (henceforth FTAP). For instance, Dalang et al. [7], Schachermayer [23], Delbaen and Schachermayer [8] or Jacod and Shiryaev [11] provide deep characterizations of the existence of martingale measures in different settings.

Partially funded by the Spanish Ministry of Science and Education (ref: BEC2003 - 09067 C04-03) and Comunidad Autónoma de Madrid (ref: s-0505/tic/000230). 
Nevertheless a simple version of the FTAP cannot be proved, in the sense that the arbitrage absence is not sufficient to construct martingale measures if the set of trading dates is not finite. It was pointed out in [2], where a simple dynamic discrete time counter-example was provided. To overcome this problem Clark [6] extended the ideas of Kreps [19] where the concept of free lunch was introduced. Under the Clark's definition the existence of free lunches is far weaker than the existence of arbitrage. The absence of free lunch has been the key to yield further extensions of the FTAP, even in the imperfect market case (see, for instance, [12]).

Any free lunch can be understood as an "approximated arbitrage" in the sense that it is "quite close" to an arbitrage portfolio. However it is almost an arbitrage but it is not an arbitrage, it is not so intuitive and its economic interpretation is not so clear. On the contrary it is introduced in mathematical terms and solves a mathematical problem, but classical pricing models (binomial model, Black and Scholes model, etc.) usually deal with the concept of arbitrage. Recent studies of efficiency in imperfect markets avoid the use of free lunches and retrieve the concept of arbitrage, but they have to deal with models containing a finite number of states of nature, case in which arbitrage strategies and free lunches coincide (see, for instance, [13]).

If feasible, it may be worth to provide risk-neutral probabilities and pricing rules (martingale measures) under simple and meaningful assumptions, as the arbitrage absence. Balbás et al. [4] have shown that it is possible to solve the counter-example of Back and Pliska [2] without drawing on free lunches. They characterize the arbitrage absence in dynamic discrete time pricing models. They build an appropriate projective system of perfect probability measures (see [20]) that are risk-neutral for each finite subset of trading dates. Then they show that the projective limit is risk-neutral for the whole set of trading dates, in the sense that the set of states of the world and the price process may be extended to a "new price process" which is a martingale under this projective limit. The initial probability measure and the risk-neutral one cannot be equivalent, as illustrated by using the counter-example of Back and Pliska [2]. However, for any finite subset of trading dates one can find projections of both measures that are equivalent, and there are Radon-Nikodym derivatives in both directions. Balbás et al. [4] use this property to introduce the concept of "projective equivalence" of probability measures.

The solution of Balbás et al. [4] allows us to prove further extensions of the FTAP that retrieve the equivalence between the initial and the martingale measure. It has been pointed out by Balbás et al. [3], where the equivalence is proved under some assumptions on the ratio "risk/return". A recent approach about risk measurement may be found in [21].

Another caveat appears when characterizing the arbitrage absence for (even static) models with an infinite family of securities. This is clearly pointed out in [23], where a simple counter-example with a countable number of assets is provided. Models with infinitely many assets may be useful when dealing with interest rates 
(see [9]) or when dealing with markets for which several parameters are not fixed (consider for instance a derivative market where call options with infinitely many strikes may be available). Moreover, as will be illustrated in Remark 4.12 (Section 4), every dynamic pricing model could be adapted in such a way that it becomes a model with infinitely many assets

The literature has addressed different properties of those models with infinitely many assets (for example, Aliprantis et al. [1] analyze the existence of some kind of equilibrium). The theory of large financial market is a quite interesting alternative that also provides martingale measures (see, for instance, [14] or [17], among others). Each "small" market is arbitrage free and there is even an equivalent martingale measure on each of the small markets. Still there can be various forms of approximate arbitrage opportunities when one considers the sequence of markets, and the notion of "no arbitrage" is generalized to be sufficient to get a risk neutral measure for the large financial market.

The approach of Balbás et al. [4] could be useful to analyze new problems related to the FTAP. For instance, imperfect markets (see, for example, [15], [16] or [24]) or markets with infinitely many assets. This paper follows this approach and addresses one-period perfect models with infinitely many securities. The study seems to be general enough since there are no assumptions on the properties of the set of securities and the notion of arbitrage is not extended.

The existence of risk-neutral probabilities will be stated by means of projective limits of projective systems of Radon probability measures (see [25]), rather than projective systems of perfect measures. These projective systems will permit us to extend the concept of projective equivalence and to broaden the set of states of nature. In some sense the new set of states of nature may be identified with the set of paths of real prices and, therefore, it better captures the price behavior. We could interpret that the failure of the FTAP partially obeys to the "insufficiency" of the set of states to explain the whole price process.

The outline of the article is as follows. Section 2 will introduce the basic concepts and notation. Section 3 will transform the problem in order to introduce the "projective system approach" and will define the notion of "projectively equivalent martingale measure". Two counter-examples will illustrate the FTAP failure, as well as the role of projectively equivalent martingale measures in order to address the caveat. The first counter-example is adapted from the one of Back and Pliska [2], although we consider only two trading dates (instead of an infinite number of them) and infinitely many securities (instead of two ones). The second one is that introduced by Schachermayer [23], though this author never used the example to deal with projective systems. Section 4 presents those results concerning the existence of projectively equivalent martingale measures. In particular, Theorem 4.1 will point out that the arbitrage absence is the necessary and sufficient condition for complete markets, whereas Theorem 4.7 and Remark 4.12 will yield some sufficient conditions for incomplete markets. The counter-example of Schachermayer [23] will prove that there are arbitrage free (incomplete) markets for which it is not feasible to yield any price of some new securities and, consequently, it is impossible 
to find projectively equivalent martingale measures. Hence there exists a critical difference between both counter-examples. The last section concludes the paper.

\section{Preliminaries and notation}

Let $(\Omega, \mathcal{F}, \mu)$ be a probability space composed of the set $\Omega$, the $\sigma$-algebra $\mathcal{F}$ and the probability measure $\mu$. Consider a set $\left(S_{i}\right)_{i \in I}$ of available securities and a second set $\left(f_{i}\right)_{i \in I} \subset L^{2}(\mu)$ of random variables providing the pay-off at a future date $T$ of $S_{i}$, for every $i \in I$. Denote by $\left(p_{i}\right)_{i \in I} \subset \mathbb{R}$ the family of current prices. Let us assume that $0 \in I$ and $S_{0}$ is a numeraire, in the sense that $p_{0}=1$ and $f_{0}=1, \mu-a . s$.

The set of feasible portfolios will be the vector space $E_{\infty}=\left\{\left(x_{i}\right)_{i \in I} \subset \mathbb{R}\right.$; there exists $J \subset I$ with $J$ finite and $x_{i}=0$ whenever $\left.i \notin J\right\}$. The current price and the future pay-off of $x=\left(x_{i}\right)_{i \in I} \in E_{\infty}$ will be given by

$$
\lambda(x)=\sum_{i \in I} x_{i} p_{i} \in \mathbb{R}
$$

and

$$
\Lambda(x)=\sum_{i \in I} x_{i} f_{i} \in L^{2}(\mu)
$$

respectively. As usual an arbitrage portfolio allows traders to get "money without risk". A risk-neutral measure makes prices be mean values of each pay-off. We have:

Definition 2.1. A portfolio $x \in E_{\infty}$ is said to be an arbitrage if $\lambda(x) \leq 0, \Lambda(x) \geq 0$ $\mu-a . s$. and $\mu(\omega \in \Omega: \Lambda(x)(\omega)-\lambda(x)>0)>0$.

Notice that those arbitrage profits obtained at the current date may by invested in the riskless asset $S_{0}$. Whence the existence of arbitrage is equivalent to the existence of self-financing arbitrage for which $\lambda(x)=0$ holds.

Definition 2.2. The $\sigma$-additive measure $\nu: \mathcal{F} \longmapsto[0,1]$ is said to be a risk-neutral probability (or a risk-neutral probability measure, or a martingale measure) if

(i) $\mu$ and $\nu$ are equivalent, i.e., $\mu(A)=0 \Longleftrightarrow \nu(A)=0$.

(ii)

$$
p_{i}=\int_{\Omega} f_{i} \mathrm{~d} \nu
$$

for every $i \in I$.

The absence of arbitrage and the FTAP guarantee the existence of riskneutral probability measures for any finite sets of securities (see, for instance, [7], [23] or [11]).

Henceforth $\mathcal{P}_{F}(I)$ will denote the set of finite subsets of $I$ containing 0 . 
Theorem 2.3. The model is arbitrage-free if and only if a net $\left(\tilde{\nu}_{J}\right)_{J \in \mathcal{P}_{F}(I)}$ of $\sigma$-additive probability measures exists on $\mathcal{F}$ such that $\mu$ and $\tilde{\nu}_{J}$ are equivalent for every $J \in \mathcal{P}_{F}(I)$ and

$$
p_{i}=\int_{\Omega} f_{i} \mathrm{~d} \tilde{\nu}_{J}
$$

whenever $J \in \mathcal{P}_{F}(I)$ and $i \in J$.

Despite the previous result, several counter-examples point out that the riskneutral measure $\tilde{\nu}_{J}$ depends on $J$, i.e., in general, it is not possible to find $\nu$ : $\mathcal{F} \longmapsto[0,1]$ satisfying the conditions of Definition 2.2. Two interesting counterexamples will be presented in the next section, where we will introduce an adequate framework that may solve this caveat.

\section{Projective system approach}

For every set $C$ we will denote by $\mathbb{R}^{C}$ the set of $\mathbb{R}$-valued functions on $C$ endowed with the usual product topology and the Borel $\sigma$-algebra $\mathcal{B}_{C}$.

Let $J \in \mathcal{P}_{F}(I)$. Consider the probability space

$$
\left(\mathbb{R}^{J}, \mathcal{B}_{J}, \mu_{J}\right)
$$

where $\mu_{J}$ is the probability measure $f_{J}(\mu)$ given by

$$
\mu_{J}(B)=\mu\left[f_{J}^{-1}(B)\right]
$$

for every $B \in \mathcal{B}_{J}, f_{J}$ being the measurable function

$$
\Omega \ni \omega \longmapsto f_{J}(\omega)=\left(f_{i}(\omega)\right)_{i \in J} \in \mathbb{R}^{J} .
$$

Then $\left(\mu_{J}\right)_{J \in \mathcal{P}_{F}(I)}$ is a projective system of Radon probability measures (see [25]), in the sense that, denoting the natural projection by

$$
\pi_{J, K}: \mathbb{R}^{K} \longmapsto \mathbb{R}^{J}
$$

we have that $\mu_{J}=\pi_{J, K}\left(\mu_{K}\right)$ whenever $J, K \in \mathcal{P}_{F}(I)$ and $J \subset K$.

For every $J \in \mathcal{P}_{F}(I)$ one can consider the one-period pricing model defined on the probability space (3.1) and generated by the finite family of securities whose current prices are $\left(p_{i}\right)_{i \in J}$ and whose pay-off are given by the natural projections

$$
\pi_{\{i\}, J}: \mathbb{R}^{J} \longmapsto \mathbb{R}
$$

$i \in J$. This new model will be called $J^{t h}$-market.

Proposition 3.1. The initial model is arbitrage free if and only if the $J^{\text {th }}$-market is arbitrage free for every $J \in \mathcal{P}_{F}(I)$.

Proof. The $J^{t h}$-market is not arbitrage free if and only if there exists a selffinancing portfolio $\left(x_{i}\right)_{i \in J}$ such that

$$
\mu_{J}\left[\left(\alpha_{i}\right)_{i \in J}: \sum_{i \in J} x_{i} \alpha_{i} \geq 0\right]=1 \text { and } \mu_{J}\left[\left(\alpha_{i}\right)_{i \in J}: \sum_{i \in J} x_{i} \alpha_{i}>0\right]>0 \text {. }
$$


This is equivalent to

$$
\mu\left[\omega \in \Omega: \sum_{i \in J} x_{i} f_{i}(\omega) \geq 0\right]=1 \quad \text { and } \quad \mu\left[\omega \in \Omega: \sum_{i \in J} x_{i} f_{i}(\omega)>0\right]>0
$$

which means that the initial model is not arbitrage free.

Assumption 1. From now on we will assume that $\left(f_{i}\right)_{i \in I} \subset L^{\infty}(\mu){ }^{1}$ set $^{2}$

Assumption 1 implies that $\mu_{J}$ has a compact support included in the compact

$$
\Pi_{i \in J}\left[-\left\|f_{i}\right\|_{\infty},\left\|f_{i}\right\|_{\infty}\right] \subset \mathbb{R}^{J}
$$

for every $J \in \mathcal{P}_{F}(I)$. Hence the Prokhorov Theorem (see [25]) guarantees the existence of a unique Radon probability measure $\mu_{I}$ on the measurable space $\left(\mathbb{R}^{I}, \mathcal{B}_{I}\right)$ that is the projective limit of the system $\left(\mu_{J}\right)_{J \in \mathcal{P}_{F}(I)}$, i.e.,

$$
\mu_{J}=\pi_{J, I}\left(\mu_{I}\right)
$$

holds for every $J \in \mathcal{P}_{F}(I)$. Moreover, $\mu_{I}$ has a compact support included in the compact set

$$
\Pi_{i \in I}\left[-\left\|f_{i}\right\|_{\infty},\left\|f_{i}\right\|_{\infty}\right] \subset \mathbb{R}^{I} .
$$

Now we can introduce a key concept for this paper.

Definition 3.2. A Radon probability measure $\nu_{I}$ on the measurable space $\left(\mathbb{R}^{I}, \mathcal{B}_{I}\right)$ is a projectively equivalent martingale measure (or a projectively equivalent riskneutral probability) if:

(i) $\mu_{I}$ and $\nu_{I}$ are projectively equivalent, i.e., $\mu_{J}$ and $\nu_{J}=\pi_{J, I}\left(\nu_{I}\right)$ are equivalent for every $J \in \mathcal{P}_{F}(I)$.

(ii) Given $J \in \mathcal{P}_{F}(I)$ we have that $\nu_{J}$ is a martingale measure for the $J^{t h}$ market. ${ }^{3}$

Despite $\mu_{I}$ and $\nu_{I}$ do not have to be equivalent notice that Condition $(i)$ above guarantees the existence of positive densities between their projections. This also implies that the compact supports of $\nu_{I}$ and its projections are included in (3.4) and (3.3) respectively.

Notice that $\Omega$ may be interpreted as a subset of $\mathbb{R}^{I}$ owing to "the immersion" (3.2) where $J$ is replaced by $I{ }^{4}$ In some sense the projective system approach allows us to enlarge the set of states of nature and to identify this set and the set of real prices.

Next let us prove a preliminary result.

\footnotetext{
${ }^{1}$ This assumption significantly simplifies the exposition. Anyway most of the theory would still hold if the assumption failed, though the the role of the Prokhorov Theorem (see [25]) should be replaced by the Daniel-Kolmogorov Theorem (see [18]).

${ }^{2}\left[-\left\|f_{0}\right\|_{\infty},\left\|f_{0}\right\|_{\infty}\right]=[-1,1]$ may be replaced by $\{1\}$. An analogous comment applies for (3.4).

$3_{i . e .,} p_{i}=\int_{\mathbb{R}^{J}} \pi_{\{i\}, J} \mathrm{~d} \nu_{J}=\int_{\mathbb{R}^{I}} \pi_{\{i\}, I} \mathrm{~d} \nu_{I}$ holds for every $J \in \mathcal{P}_{F}(I)$ and every $i \in J$.

${ }^{4}$ This immersion is not necessarily measurable, although this problem is solved if we consider the cylindrical $\sigma$-algebra of $\mathbb{R}^{I}$ instead of the Borel one (see [18]).
} 
Proposition 3.3. Statements below satisfy the implications 3.3.1) $\Rightarrow 3.3 .2) \Leftrightarrow$ 3.3.3) $\Rightarrow 3.3 .4$ ).

3.3.1) There exists a martingale measure $\nu$.

3.3.2) There exists a projective system $\left[\nu_{J}\right]_{J \in \mathcal{P}_{F}(I)}$ of Radon measures such that $\nu_{J}$ is a martingale measure for the $J^{\text {th }}$-market.

3.3.3) There exists a projectively equivalent martingale measure $\nu_{I}$.

3.3.4) The initial model is arbitrage free.

Proof. $[3.3 .1) \Rightarrow 3.3 .2)]$. Given $J \in \mathcal{P}_{F}(I)$ take $\nu_{J}=f_{J}(\nu)$, where $f_{J}$ is represented in (3.2). Then the equivalence between $\mu$ and $\nu$ trivially leads to the equivalence between $\mu_{J}$ and $\nu_{J}$, and the equality

$$
p_{i}=\int_{\mathbb{R}^{J}} \pi_{\{i\}, J} \mathrm{~d} \nu_{J}
$$

for $i \in J$, trivially follows from (2.1). Finally, if $J, K \in \mathcal{P}_{F}(I)$ and $J \subset K$, then $\nu_{J}=f_{J}(\nu)=\pi_{J, K} f_{K}(\nu)=\pi_{J, K}\left(\nu_{K}\right)$.

$[3.3 .2) \Rightarrow 3.3 .3)]$. Since any $\nu_{J}$ is equivalent to $\mu_{J}$ their supports are included in the compact sets (3.3). Thus the Prokhorov Theorem ensures the existence of the projective limit $\nu_{I}$.

$[3.3 .3) \Rightarrow 3.3 .2)]$. Just define $\nu_{J}=\pi_{J, I}\left(\nu_{I}\right)$ for every $J \in \mathcal{P}_{F}(I)$.

$[3.3 .2) \Rightarrow 3.3 .4)]$. Theorem 2.3 ensures that the $J^{\text {th }}$-market is arbitrage-free so 3.3 .4$)$ trivially follows from Proposition 3.1.

Examples 1 and 2 below will be clear counter-examples showing that, in general, 3.3.2) $\nRightarrow 3.3 .1)$ and 3.3.4) $\nRightarrow 3.3 .2$ ).

Example 1. Consider $I=\{0,1,2, \ldots\}=\mathbb{N}, \Omega=\{1,2, \ldots\}=\mathbb{N} \backslash\{0\}, \mathcal{F}$ the discrete $\sigma$-algebra of $\Omega$ and suppose that $\mu(\omega)>0$ for every $\omega \in \Omega$. Let be $p_{i}=1$, $i=0,1,2, \ldots$ and

$$
f_{i}(\omega)= \begin{cases}\frac{\omega^{2}+2 \omega+2}{2^{\omega}} & \omega \leq i \\ \frac{1}{2^{i}} & \omega>i\end{cases}
$$

$i, \omega=1,2, \ldots$ To make it easy, let us provide the infinite matrix below whose $i^{\text {th }}$-column reflects the pay-off of $S_{i}, i=0,1,2, \ldots$

$$
\mathcal{M}_{1}=\left(\begin{array}{llllll}
1 & 5 / 2 & 5 / 2 & 5 / 2 & 5 / 2 & \ldots \\
1 & 1 / 2 & 10 / 4 & 10 / 4 & 10 / 4 & \ldots \\
1 & 1 / 2 & 1 / 4 & 17 / 8 & 17 / 8 & \ldots \\
1 & 1 / 2 & 1 / 4 & 1 / 8 & 26 / 16 & \ldots \\
\ldots & \ldots & \ldots & \ldots & \ldots & \ldots
\end{array}\right) .
$$

Consider $i, \omega \in \Omega$ such that $\omega>i$ and define $\nu_{i, \omega}>0$ so that

$$
\sum_{\omega=i+1}^{\infty} \nu_{i, \omega}=1-\sum_{\omega=1}^{i} \frac{1}{2 \omega(\omega+1)}=\frac{i+2}{2 i+2}
$$


holds. Clearly, the existence of $\left(\nu_{n, \omega}\right)_{\omega=n+1}^{\infty}$ is guaranteed for every $n \in \Omega=\mathbb{N} \backslash\{0\}$. Take $n \in \Omega, J_{n}=\{0,1, \ldots, n\}$, and define

$$
\tilde{\nu}_{n}(\omega)= \begin{cases}\frac{1}{2 \omega(\omega+1)} & \omega \leq n \\ \nu_{n, \omega} & \omega>n .\end{cases}
$$

It can be easily proved that $\mu$ and $\tilde{\nu}_{n}$ are equivalent for $n=1,2, \ldots$ and condition (2.2) is satisfied whenever $i \in J_{n}$. Therefore Theorem 2.3 ensures that the market is arbitrage free. However, it can be observed that a risk-neutral probability measure $\nu$ as in Definition 2.2 does not exist. Indeed, if it existed we would obtain by induction

$$
\nu(\omega)=\frac{1}{2 \omega(\omega+1)}
$$

but then $\sum_{\omega=1}^{\infty} \nu(\omega)=\frac{1}{2}$ and $\sum_{\omega=1}^{\infty} f_{i}(\omega) \nu(\omega)<1$ for all $i=0,1, \ldots$

Next let us build the projectively equivalent martingale measure that illustrates the fulfillment of 3.3.3).

Notice that the rows of $\mathcal{M}_{1}$ provide the measure $\mu_{J_{n}}$ associated with the $J_{n}^{t h}$-market. Indeed, it is easy to see that

$$
\begin{aligned}
& \left.\mu_{J_{n}}(1,5 / 2,5 / 2, \ldots, 5 / 2)\right)=\mu(1) \\
& \mu_{J_{n}}(1,1 / 2,10 / 4, \ldots 10 / 4)=\mu(2) \\
& \mu_{J_{n}}(1,1 / 2,1 / 4,17 / 8, \ldots 17 / 8)=\mu(3) \\
& \mu_{J_{n}}\left(1, f_{1}(n), f_{2}(n), \ldots \ldots, f_{n}(n)\right)=\mu(n) \\
& \mu_{J_{n}}\left(1, f_{1}(n+1), f_{2}(n+1), \ldots \ldots, f_{n}(n+1)\right)=\sum_{r=n+1}^{\infty} \mu(r) .
\end{aligned}
$$

It is important to point out that the $J_{n}^{\text {th }}$-market is complete, in the sense that it involves $n+1$ independent securities and the support of $\mu_{J_{n}}$ contains $n+1$ points of $\mathbb{R}^{J_{n}}$. Therefore the risk-neutral probability of this market is unique and it is easy to see that it is given by

$$
\begin{gathered}
\left.\nu_{J_{n}}(1,5 / 2,5 / 2, \ldots, 5 / 2)\right)=\nu(1) \\
\nu_{J_{n}}(1,1 / 2,10 / 4, \ldots 10 / 4)=\nu(2) \\
\nu_{J_{n}}(1,1 / 2,1 / 4,17 / 8, \ldots 17 / 8)=\nu(3) \\
\ldots \ldots \ldots \ldots \ldots \ldots \ldots \ldots . . \\
\nu_{J_{n}}\left(1, f_{1}(n), f_{2}(n), \ldots \ldots, f_{n}(n)\right)=\nu(n) \\
\nu_{J_{n}}\left(1, f_{1}(n+1), f_{2}(n+1), \ldots \ldots, f_{n}(n+1)\right)=\sum_{\omega=n+1}^{\infty} \nu_{n, \omega}
\end{gathered}
$$

where $\nu$ and $\sum_{\omega=n+1}^{\infty} \nu_{n, \omega}$ are given in (3.6) and (3.5) respectively. In order to see that 3.3.2) or 3.3.3) are fulfilled it is sufficient to prove that

$$
\pi_{J_{n}, J_{n+1}}\left(\nu_{J_{n+1}}\right)=\nu_{J_{n}},
$$


$n=1,2, \ldots$, but this trivially follows from equalities above. Finally, the previous projective system clearly converges to the measure $\nu_{\mathbb{N}}$ whose support is concentrated in the rows of $\mathcal{M}_{1}$ plus the additional sequence

$$
\left(1, \frac{1}{2}, \frac{1}{4}, \ldots\right)=\left(\frac{1}{2^{n}}\right)_{n=0}^{\infty}
$$

Furthermore, $\nu(\omega)$ (see (3.6)) coincides with $\nu_{\mathbb{N}}$ on the $\omega^{t h}$-row of $\mathcal{M}_{1}, \omega=1,2, \ldots$, and

$$
\nu_{\mathbb{N}}\left(\frac{1}{2^{n}}\right)_{n=0}^{\infty}=\frac{1}{2} .
$$

Overall, as already said in the introduction, the projective system approach allows us to enlarge the set of states of nature and to identify this set and the set of paths of real prices, since (3.7) is reflecting "the only trajectory of prices not contained in the columns of $\mathcal{M}_{1} "$.

Example 2. Next let us introduce a new example illustrating that 3.3.4) $\nRightarrow 3.3 .2$ ) or 3.3.3). Consider again $I=\mathbb{N}, \Omega=\mathbb{N} \backslash\{0\}, \mathcal{F}$ the discrete $\sigma$-algebra of $\Omega$ and suppose that $\mu(\omega)>0$ for every $\omega \in \Omega$. Let be $p_{i}=0, i=1,2, \ldots$ and $f_{i}(i)=1$, $f_{i}(i+1)=-1$ and $f_{i}(\omega)=0$ for every $i, \omega=1,2, \ldots$ with $\omega \neq i$ and $\omega \neq i+1$. As in Example 1, it may be convenient to provide the pay-off matrix

If we define

$$
\mathcal{M}_{2}=\left(\begin{array}{llllll}
1 & 1 & 0 & 0 & 0 & \ldots \\
1 & -1 & 1 & 0 & 0 & \ldots \\
1 & 0 & -1 & 1 & 0 & \ldots \\
1 & 0 & 0 & -1 & 1 & \ldots \\
\ldots & \ldots & \ldots & \ldots & \ldots & \ldots
\end{array}\right) .
$$

for $n=1,2, \ldots$ and $\omega=1,2, \ldots, n, n+1$, and

$$
\tilde{\nu}_{n}(\omega)=\frac{1}{2(n+1)}
$$

$$
\tilde{\nu}_{n}(\omega)=\frac{\mu(\omega)}{2 \sum_{\omega^{*}=n+2}^{\infty} \mu\left(\omega^{*}\right)}
$$

for $n=1,2, \ldots$ and $\omega=n+2, n+3, \ldots$, then it may be easily proved that Theorem 2.3 holds and therefore the market is arbitrage free. Besides, according to (2.1), a risk-neutral probability $\nu$ should satisfy

$$
0<\nu(1)=\nu(2)=\nu(3)=\ldots
$$

which makes it impossible to verify $\nu(1)+\nu(2)+\nu(3)+\ldots=1$. Furthermore, as in the previous case the rows of $\mathcal{M}_{2}$ yield the measure $\mu_{J_{n}}$ of the projective system approach. Indeed,

$$
\begin{gathered}
\mu_{J_{n}}(1,1,0, \ldots, 0)=\mu(1) \\
\mu_{J_{n}}(1,-1,1,0, \ldots, 0)=\mu(2) \\
\mu_{J_{n}}(1,0,-1,1,0, \ldots, 0)=\mu(3) \\
\mu_{J^{n}}(1,0,0,-1,1,0, \ldots, 0)=\mu(4)
\end{gathered}
$$




$$
\begin{aligned}
& \mu_{J_{n}}(1,0, \ldots, 0,-1)=\mu(n+1) \\
& \mu_{J_{n}}(1,0, \ldots, 0)=\sum_{r=n+2}^{\infty} \mu(r) .
\end{aligned}
$$

Note that there is an important difference between both examples since the $J_{n}^{t h}$-market is not complete. In fact, the number of states equals $n+2$ while the number of securities equals $n+1$. Accordingly, the number of risk-neutral measures for this market is infinite. So, if $\Lambda_{n}$ denotes the set of risk-neutral measures then each element of $\Lambda_{n}$ is characterized by two strictly positive parameters $\lambda$ and $\lambda^{*}$ such that

$$
(n+1) \lambda+\lambda^{*}=1 .
$$

Thus the corresponding risk-neutral measure satisfies

$$
\begin{aligned}
\nu_{J_{n}}^{\lambda}(1,1,0, \ldots, 0)=\nu_{J_{n}}^{\lambda}(1,-1,1,0, \ldots, 0) & =\nu_{J_{n}}^{\lambda}(1,0,0,-1,1,0, \ldots, 0) \ldots \\
& =\nu_{J_{n}}^{\lambda}(1,0, \ldots, 0,-1)=\lambda
\end{aligned}
$$

and

$$
\nu_{J_{n}}^{\lambda}(1,0, \ldots, 0)=\lambda^{*} .
$$

In order to show that 3.3.2) fails suppose that $\left(\nu_{J_{n}}^{\lambda_{n}}\right)_{n=1}^{\infty}$ is a projective system satisfying the conditions of 3.3.2). Fix $n \in \mathbb{N} \backslash\{0\}$. One has that

$$
\pi_{J_{n}, J_{n+m}}\left(\nu_{J_{n+m}}^{\lambda_{n+m}}\right)=\nu_{J_{n}}^{\lambda_{n}}
$$

for every $m \in \mathbb{N}$, from where

$$
\lambda_{n}=\nu_{J_{n}}^{\lambda_{n}}(1,1,0, \ldots, 0)=\nu_{J_{n+m}}^{\lambda_{n+m}}(1,1,0, \ldots, 0)=\lambda_{n+m} .
$$

From (3.8) one has that

$$
\lambda_{n}=\lambda_{n+m}<\frac{1}{n+m+1}
$$

and therefore, taking $m \longmapsto \infty$, we have $\lambda_{n}=0$. But this contradicts the equivalence between $\mu_{J_{n}}$ and $\nu_{J_{n}}^{\lambda_{n}}$.

\section{Existence of projectively equivalent martingale measures}

Next let us introduce a first result justifying the success of the Projective System Approach in Example 1. In addition it will illustrate the utility of projectively equivalent martingale measures when pricing new assets.

Theorem 4.1. Suppose that there exists a cofinal subset $\mathcal{C} \subset \mathcal{P}_{F}(I)$ such that the $J^{\text {th }}$-market is complete for every $J \in \mathcal{C}$. Then Assertions 3.3.2), 3.3.3) and 3.3.4) are equivalent. Furthermore, in the affirmative case the following properties hold:

4.1.1) The projectively equivalent martingale measure $\nu_{I}$ is unique. 
4.1.2) Consider $J \in \mathcal{P}_{F}(I), \nu_{J}=\pi_{J, I}\left(\nu_{I}\right), \varphi \in L^{\infty}\left(\mathbb{R}^{J}, \mathcal{B}_{J}, \mu_{J}\right)$ and the new security $S_{\varphi}$ whose pay-off at $T$ is given by

$$
f_{\varphi}=\varphi \circ f_{J} \in L^{\infty}(\mu) .
$$

One has that

$$
p_{\varphi}=\int_{\mathbb{R}^{I}}\left(\varphi \circ \pi_{J, I}\right) \mathrm{d} \nu_{I}
$$

is the only price of $S_{\varphi}$ making the market $\left(S_{i}\right)_{i \in I} \cup\left(S_{\varphi}\right)$ arbitrage free.

Proof. Suppose that 3.3.4) holds. Take $K \in \mathcal{C}$. Proposition 3.1 and the completeness of the $K^{t h}$-market ensure the existence of $\nu_{K}$, unique martingale measure for the $K^{t h}$-market. If $J \notin \mathcal{C}$ consider $K \in \mathcal{C}$ with $J \subset K$ and set

$$
\nu_{J}=\pi_{J, K}\left(\nu_{K}\right) .
$$

It is clear that $\nu_{J}$ does not depend on $K$. Indeed, if $K^{\prime} \in \mathcal{C}$ and $J \subset K^{\prime}$ then take $K^{*} \supset K \cup K^{\prime}$ such that $K^{*} \in \mathcal{C}$ and we have that

$$
\nu_{K}=\pi_{K, K^{*}}\left(\nu_{K^{*}}\right)
$$

holds due to the uniqueness of the martingale measure for the $K^{t h}$-market. Analogously

from where

$$
\nu_{K^{\prime}}=\pi_{K^{\prime}, K^{*}}\left(\nu_{K^{*}}\right)
$$

$$
\pi_{J, K}\left(\nu_{K}\right)=\pi_{J, K} \pi_{K, K^{*}}\left(\nu_{K^{*}}\right)=\pi_{J, K^{*}}\left(\nu_{K^{*}}\right)=\pi_{J, K^{\prime}} \pi_{K^{\prime}, K^{*}}\left(\nu_{K^{*}}\right)=\pi_{J, K^{\prime}}\left(\nu_{K^{\prime}}\right) .
$$

In order to see that $\left(\nu_{J}\right)_{J \in \mathcal{P}_{F}(I)}$ is a projective system it is sufficient to bear in mind (4.3) and (4.4). Thus, 3.3.2) holds.

In order to prove 4.1.1) it is sufficient to realize that the projections of $\nu_{I}$ are unique on a cofinal subset $\mathcal{C}$. Consequently the projections are unique on the whole set $\mathcal{P}_{F}(I)$ and the uniqueness of $\nu_{I}$ trivially follows from the uniqueness of the projective limit of projective systems of Radon measures (see [25]).

Finally, to prove 4.1.2), consider the security above $S_{\varphi}$. As in the proof of Proposition 3.1 one can establish that the market $\left(S_{i}\right)_{i \in I} \cup\left(S_{\varphi}\right)$ is arbitrage free if and only if for every $K \in \mathcal{P}_{F}(I)$ with $K \supset J$ the market

$$
\left(\pi_{\{i\}, K}\right)_{i \in K} \cup\left(\varphi \circ \pi_{J, K}\right)
$$

is arbitrage free. In particular if this holds and $K \in \mathcal{C}$ the uniqueness of $\pi_{K, I}\left(\nu_{I}\right)$ leads to (4.2). Conversely (4.2) guarantees that (4.5) is arbitrage free for every $K \in \mathcal{C}$ and, therefore, for every $K \in \mathcal{P}_{F}(I)$.

The latter theorem and Expression (4.2) point out that projectively equivalent risk-neutral probabilities may yield pricing rules that enable us to value new securities of complete markets. So it is worth to illustrate that the valuation of new securities is not always feasible for incomplete markets. Additionally this also anticipates some intuitions about the reasons of the Projective System Approach failure when dealing with Example 2. 
Remark 4.2. Consider the market of Example 2 plus a new asset $S_{\varphi}$ whose pay-off at $T$ is given by

$$
f_{\varphi}=\left(2 f_{1}-1\right)^{+}= \begin{cases}1, & \omega=1 \\ 0, & \omega \neq 1\end{cases}
$$

Obviously $S_{\varphi}$ may be understood as a call option with expiration at $T$, strike equal to one monetary unit and underlying asset composed of two units of $S_{1}$. It is also easy to check that

$$
f_{\varphi}=\varphi \circ f_{\{0,1\}}
$$

if

$$
\varphi: \mathbb{R}^{\{0,1\}} \longrightarrow \mathbb{R}
$$

is given by

$$
\varphi(x, y)=(2 y-1)^{+},
$$

so $S_{\varphi}$ has the general form proposed in (4.1).

Next let us prove that it is impossible to provide $S_{\varphi}$ with a price $p_{\varphi} \in \mathbb{R}$ unless we accept the existence of arbitrage. First, $\mu\left(f_{\varphi} \geq 0\right)=1$ and $\mu\left(f_{\varphi}>\right.$ $0)>0$, along with the arbitrage absence, will imply that $p_{\varphi}>0$. Second, if the market $\left(S_{n}\right)_{n \in \mathbb{N}} \cup\left(S_{\varphi}\right)$ is arbitrage-free then (see Definition 2.1) the market $\left\{S_{0}, S_{1}, \ldots, S_{m}, S_{\varphi}\right\}$ is arbitrage-free for every $m \in \mathbb{N}$. Choose $m$ such that

$$
\frac{1}{m+1}<p_{\varphi}
$$

Then it is easy to show that every risk-neutral measure $\tilde{\nu}_{m}$ for the market $\left\{S_{0}, S_{1}, \ldots, S_{m}\right\}$ will satisfy

$$
\tilde{\nu}_{m}(1) \leq \frac{1}{m+1}
$$

from where the price $p_{\varphi}$ does not prevent the existence of arbitrage.

Theorem 4.7 and Remark 4.12 below will present some general models for which the implication 3.3.4) $\Rightarrow$ 3.3.3) holds. Moreover it shows that projectively equivalent risk-neutral probabilities, if they exist, provide us with pricing rules for incomplete markets as well. Before presenting their statements we need some additional concepts.

Definition 4.3. Suppose that the initial model is arbitrage free. We will say that the $P$-property holds if for every $J \in \mathcal{P}_{F}(I)$ and every $\varphi \in L^{\infty}\left(\mathbb{R}^{J}, \mathcal{B}_{J}, \mu_{J}\right)$, the new security $S_{\varphi}$ whose pay-off at $T$ is given by $f_{\varphi}=\varphi \circ f_{J} \in L^{\infty}(\mu)$ has at least one price $p_{\varphi} \in \mathbb{R}$ making the market $\left(S_{i}\right)_{i \in I} \cup\left(S_{\varphi}\right)$ arbitrage free.

Definition 4.4. Suppose that the initial model is arbitrage free, and for every $J \in$ $\mathcal{P}_{F}(I)$ consider the set $\mathcal{R}_{J}$ of martingale measures for the $J^{t h}$-market. Proposition 3.1 guarantees that each $\mathcal{R}_{J}$ is non-empty. We will say that the ${ }^{*}$-property holds if there exists a cofinal subset $\mathcal{C} \subset \mathcal{P}_{F}(I)$ such that $\mathcal{R}_{J}$ is uniformly $\mu_{J}$-continuous for every $J \in \mathcal{C}$, i.e., for every $J \in \mathcal{C}$ and every $\varepsilon>0$ there exists $\delta>0$ such that 
the implication

$$
B_{J} \in \mathcal{B}_{J} \text { and } \mu_{J}\left(B_{J}\right) \leq \delta \Longrightarrow \theta_{J}\left(B_{J}\right) \leq \varepsilon, \text { for every } \theta_{J} \in \mathcal{R}_{J}
$$

holds.

Definition 4.5. We will say that the initial model verifies the ${ }^{* *}$-property if there exists a cofinal subset $\mathcal{C} \subset \mathcal{P}_{F}(I)$ such that for every $J \in \mathcal{C}$ and every compact set $X_{J} \subset \mathbb{R}^{J}$ with void interior and positive probability $\left(\mu_{J}\left(X_{J}\right)>0\right)$ there exists a $\mu_{J}-$ atom $Y_{J}$ with positive probability and such that $Y_{J} \subset X_{J}$.

Remark 4.6. The ${ }^{*}$-property holds for many interesting cases. For instance, it is obviously fulfilled for complete markets since $\mathcal{R}_{J}$ is a singleton. It is easy to see that it is also fulfilled if for any $\mu_{J}$ (or a cofinal family) there exists a finite and disjoint collection of $\mu_{J}$-atoms

$$
B_{J}^{1}, B_{J}^{2}, \ldots B_{J}^{r}
$$

( $r$ depending on $J$ ) such that

$$
\sum_{s=1}^{r} \mu_{J}\left(B_{J}^{s}\right)=1
$$

In particular, the model of Example 2 satisfies the ${ }^{*}$-property.

Analogously, The ${ }^{* *}$ - property also holds for many interesting cases like complete markets or Example 2. More generally, it is easy to prove that the property holds if any $\mathbb{R}^{J}$ (or a cofinal family) may be divided into a countable and disjoint collection of $\mu_{J}$-atoms.

Theorem 4.7. 4.7.1) If there exists $\nu_{I}$, projectively equivalent martingale measure, then the initial model is arbitrage-free and satisfies the P-property. Furthermore, (4.2) is a price of (4.1) making the market $\left(S_{i}\right)_{i \in I} \cup\left(S_{\varphi}\right)$ arbitrage free.

4.7.2) Suppose that I is countable. If the initial model is arbitrage free and satisfies the $P$-property, the ${ }^{*}$ - property and the ${ }^{* *}$-property, then there exists a projectively equivalent martingale measure.

In order to prove Theorem 4.7 we will need some technical results. Some of them may also have special interest by themselves.

Lemma 4.8. Suppose that the market is arbitrage free. Then there exists a projective system $\left(\lambda_{J}\right)_{J \in \mathcal{P}_{F}(I)}$ of Radon probability measures such that

4.8.1) The support of $\lambda_{J}$ is contained in (3.3) for every $J \in \mathcal{P}_{F}(I)$.

4.8.2) If $J \in \mathcal{P}_{F}(I)$ then $p_{i}=\int_{\mathbb{R}^{J}} \pi_{\{i\}, J} \mathrm{~d} \lambda_{J}$ for every $i \in J$.

4.8.3) If the market satisfies the $P$-property, $J \in \mathcal{P}_{F}(I)$ and $B_{J} \subset \mathbb{R}^{J}$ is a Borel set such that $\mu_{J}\left(B_{J}\right) \neq 0$, then the projective system $\left(\lambda_{K}\right)_{K \in \mathcal{P}_{F}(I)}$ may be constructed in such a way that $\lambda_{J}\left(B_{J}\right) \neq 0$.

4.8.4) If the market satisfies the ${ }^{*}$-property then $\lambda_{J}$ is $\mu_{J}$-continuous for every $J \in \mathcal{P}_{F}(I)$. 
Proof. For $J \in \mathcal{P}_{F}(I)$ we will consider the compact set $C_{J}$ given by (3.3). In addition $\mathcal{R}_{J}^{*}$ will denote the set of Radon probability measures on the Borel $\sigma$-algebra of $C_{J}$, and $\mathcal{R}_{J}$ will be composed of those $\rho_{J} \in \mathcal{R}_{J}^{*}$ such that $\rho_{J}$ and $\mu_{J}$ are equivalent and

$$
p_{i}=\int_{C_{J}} \pi_{\{i\}, J} \mathrm{~d} \rho_{J}
$$

for every $i \in J$. The absence of arbitrage and Proposition 3.1 imply that $\mathcal{R}_{J}$ is non-void.

On the other hand, the Riesz Representation Theorem allows us to identify the space $\mathcal{C}^{*}\left(C_{J}\right)$ of Radon (non necessarily positive) measures on $C_{J}$ with the dual of $\mathcal{C}\left(C_{J}\right)$, space of continuous functions on $C_{J}$, and the Alaoglu Theorem guarantees that $\mathcal{R}_{J}^{*}$ is weak $k^{*}$-compact since this set is obviously weak $k^{*}$-closed in the unit ball of $\mathcal{C}^{*}\left(C_{J}\right)$. Consequently, the Tijonov Theorem leads to the compactness of

Fix the element

$$
\mathcal{R}^{*}=\Pi_{J \in \mathcal{P}_{F}(I)} \mathcal{R}_{J}^{*}
$$

$$
\left(\rho_{J}\right)_{J \in \mathcal{P}_{F}(I)} \in \mathcal{R}^{*}
$$

in such a way that

$$
\rho_{J} \in \mathcal{R}_{J}
$$

for every $J \in \mathcal{P}_{F}(I)$. Given $J, H \in \mathcal{P}_{F}(I)$ denote $J^{c}=I \backslash J$ and consider

$$
\lambda_{J}^{H}=\pi_{J \cap H, J}\left(\rho_{J}\right) \otimes \mu_{J^{c} \cap H},
$$

where $\otimes$ is used to denote the usual tensor product of Radon measures (see [25]). ${ }^{5}$ Then it is easy to see that $\lambda_{J}^{H}$ and $\mu_{H}$ are equivalent.

For every $J \in \mathcal{P}_{F}(I)$ consider the element

$$
\Lambda_{J}=\left(\lambda_{J}^{H}\right)_{H \in \mathcal{P}_{F}(I)} \in \mathcal{R}^{*} .
$$

The compactness of $\mathcal{R}^{*}$ implies the existence of

$$
\left(\lambda_{J}\right)_{J \in \mathcal{P}_{F}(I)} \in \mathcal{R}^{*}
$$

agglomeration point of the net $\left(\Lambda_{J}\right)_{J \in \mathcal{P}_{F}(I)} \subset \mathcal{R}^{*}$.

In order to see that $\left(\lambda_{J}\right)_{J \in \mathcal{P}_{F}(I)}$ is a projective system, let us consider $J, K \in$ $\mathcal{P}_{F}(I)$ with $J \subset K$. Clearly

$$
\left(\lambda_{J}, \lambda_{K}\right) \in \mathcal{R}_{J}^{*} \times \mathcal{R}_{K}^{*}
$$

is an agglomeration point of the net

$$
\left(\lambda_{H}^{J}, \lambda_{H}^{K}\right)_{H \supset K}=\left(\pi_{J, H}\left(\rho_{H}\right), \pi_{K, H}\left(\rho_{H}\right)\right)_{H \supset K} \subset \mathcal{R}_{J}^{*} \times \mathcal{R}_{K}^{*} .
$$

Therefore (4.9) is agglomeration point of

$$
\left(\pi_{J, K} \pi_{K, H}\left(\rho_{H}\right), \pi_{K, H}\left(\rho_{H}\right)\right)_{H \supset K}
$$

and the continuity of

$$
\mathcal{R}_{K}^{*} \ni \alpha \longrightarrow \pi_{J, K}(\alpha) \in \mathcal{R}_{J}^{*}
$$

$\overline{{ }^{5} \text { Obviously } \lambda_{J}^{H}}=\pi_{H, J}\left(\rho_{J}\right)$ whenever $H \subset J$ and $\lambda_{J}^{H}=\mu_{H}$ if $H \subset J^{c}$. 
(when both spaces are endowed with the weak $k^{*}$-topology) leads to

$$
\lambda_{J}=\pi_{J, K}\left(\lambda_{K}\right) .
$$

Let us prove 4.8.2. Consider $J \in \mathcal{P}_{F}(I)$ and $i \in J$. It is clear that $\lambda_{J}$ is an agglomeration point of

$$
\left(\lambda_{H}^{J}\right)_{H \supset J}=\left(\pi_{J, H}\left(\rho_{H}\right)\right)_{H \supset J}
$$

from where the continuity of $\pi_{\{i\}, J}: \mathbb{R}^{J} \longrightarrow \mathbb{R}$ implies that

$$
\int_{\mathbb{R}^{J}} \pi_{\{i\}, J} \mathrm{~d} \lambda_{J}=\int_{\mathrm{C}_{J}} \pi_{\{i\}, J} \mathrm{~d} \lambda_{J}
$$

is an agglomeration point of

$$
\left(\int_{\mathrm{C}_{J}} \pi_{\{i\}, J} \mathrm{~d}\left(\pi_{J, H}\left(\rho_{H}\right)\right)\right)_{H \supset J}=\left(\int_{\mathrm{C}_{H}} \pi_{\{i\}, H} \mathrm{~d} \rho_{H}\right)_{H \supset J}=\left(p_{i}\right)_{H \supset J}
$$

due to (4.6) and (4.8).

Let us prove 4.8.3. Take $J \in \mathcal{P}_{F}(I)$ and the Borel set $B_{J} \subset \mathbb{R}^{J}$ such that $\mu_{J}\left(B_{J}\right)>0$. Since $\mu_{J}$ is a Radon measure with support in $C_{J}$ there exists a compact set $\tilde{C}_{J} \subset B_{J} \cap C_{J}$ with $\mu_{J}\left(\tilde{C}_{J}\right)>0$. We will prove that $\lambda_{J}\left(\tilde{C}_{J}\right)>0$. Add the new security $S_{\varphi}$ whose final pay-off is $f_{J} \circ 1_{\tilde{C}_{J}}$,

$$
1_{\tilde{C}_{J}}=\left\{\begin{array}{l}
1 \text { if } \omega \in \tilde{C}_{J} \\
0 \text { if } \omega \notin \tilde{C}_{J}
\end{array}\right.
$$

being the characteristic function of $\tilde{C}_{J}$. The $P$-property implies the existence of one (maybe non-unique) price $p_{\varphi}>0$ making the new market arbitrage free. Thus, as in the proof of Proposition 3.1, for every $H \supset J$ the $H^{t h}$-market is still arbitrage free if we add the pay-off

$$
1_{\pi_{J, H}^{-1}\left(\tilde{C}_{J}\right) \cap C_{H}}
$$

with price $p_{\varphi}$. Consequently, there are martingale measures for this new market, i.e., (4.7) may be chosen in such a way that (4.8) and $\rho_{H}\left(\pi_{J, H}^{-1}\left(\tilde{C}_{J}\right) \cap C_{H}\right)=p_{\varphi}$, for every $H \supset J$. Then

$$
\lambda_{H}^{J}\left(\tilde{C}_{J}\right)=p_{\varphi}
$$

for every $H \supset J$. Take $\varepsilon>0$ such that $p_{\varphi}-\varepsilon>0$. Since $\lambda_{J}$ is a Radon measure there exists an open set $G_{J}$ such that

$$
G_{J} \cap C_{J} \supset \tilde{C}_{J}
$$

and

$$
\lambda_{J}\left(\left(G_{J} \cap C_{J}\right) \backslash \tilde{C}_{J}\right) \leq \varepsilon .
$$

The Uryson Lemma guarantees the existence of a continuous function $h: C_{J} \longrightarrow$ $[0,1]$ that equals one on $\tilde{C}_{J}$ and vanishes on $C_{J} \backslash G_{J}$. If $H \supset J$,

$$
\int_{C_{J}} h \mathrm{~d} \lambda_{H}^{J} \geq \lambda_{H}^{J}\left(\tilde{C}_{J}\right)=p_{\varphi} .
$$


Hence, $\int_{C_{J}} h \mathrm{~d} \lambda_{J}$, agglomeration point of $\left(\int_{C_{J}} h \mathrm{~d} \lambda_{H}^{J}\right)_{H \supset J}$, satisfies

$$
\int_{C_{J}} h \mathrm{~d} \lambda_{J} \geq p_{\varphi}
$$

Thus

$$
\begin{aligned}
\lambda_{J}\left(\tilde{C}_{J}\right)=\int_{\tilde{C}_{J}} h \mathrm{~d} \lambda_{J} & =\int_{C_{J}} h \mathrm{~d} \lambda_{J}-\int_{C_{J} \backslash G_{J}} h \mathrm{~d} \lambda_{J}-\int_{\left.\left(G_{J} \cap C_{J}\right) \backslash \tilde{C}_{J}\right)} h \mathrm{~d} \lambda_{J} \\
& \geq p_{\varphi}-\varepsilon>0 .
\end{aligned}
$$

Finally, to prove 4.8 .4 , denote by $\mathcal{C}$ the cofinal subset of $\mathcal{P}_{F}(I)$ whose existence follows from the ${ }^{*}$-property. Suppose that $J \in \mathcal{P}_{F}(I)$ and $B_{J} \subset \mathbb{R}^{J}$ is a Borel set such that $\mu_{J}\left(B_{J}\right)=0$. We must prove that $\lambda_{J}$ vanishes on $B_{J}$ but, being $\lambda_{J}$ a Radon measure, we can assume that $B_{J}$ is closed. Furthermore, (4.10) allows us to assume that $J \in \mathcal{C}$.

Fix $\varepsilon>0$. Since $\pi_{J, H}\left(\rho_{H}\right), H \supset J$, are uniformly regular owing to (4.8) and the * ${ }^{*}$ property, one can take a compact set $\tilde{C}_{J} \subset C_{J} \backslash B_{J}$ such that

$$
\pi_{J, H}\left(\rho_{H}\right)\left(\left(C_{J} \backslash B_{J}\right) \backslash \tilde{C}_{J}\right) \leq \varepsilon
$$

for every $H \supset J$. If $C_{J} \cap B_{J}$ is non void then the Uryson Lemma guarantees the existence of $h: C_{J} \longrightarrow[0,1]$ continuous and such that $h$ vanishes on $\tilde{C}_{J}$ and equals one on $C_{J} \cap B_{J}$. For every $H \supset J$ one has that

$$
\pi_{J, H}\left(\rho_{H}\right)\left(C_{J} \cap B_{J}\right)=0 .
$$

Hence

$$
\begin{aligned}
0 \leq \int_{C_{J}} h \mathrm{~d} \lambda_{H}^{J} & =\int_{\left(C_{J} \backslash B_{J}\right) \backslash \tilde{C}_{J}} h \mathrm{~d} \lambda_{H}^{J}+\int_{C_{J} \cap B_{J}} h \mathrm{~d} \lambda_{H}^{J} \\
& \leq \varepsilon+\int_{C_{J} \cap B_{J}} h \mathrm{~d}\left(\pi_{J, H}\left(\rho_{H}\right)\right)=\varepsilon .
\end{aligned}
$$

Whence

$$
0 \leq \lambda_{J}\left(C_{J} \cap B_{J}\right) \leq \int_{C_{J}} h \mathrm{~d} \lambda_{J} \leq \varepsilon
$$

because $\int_{C_{J}} h \mathrm{~d} \lambda_{J}$ is agglomeration point of $\left(\int_{C_{J}} h \mathrm{~d} \lambda_{H}^{J}\right)_{H \supset J}$.

Consequently, $\lambda_{J}\left(C_{J} \cap B_{J}\right)=0$ because $\varepsilon$ can take any positive value. ${ }^{6}$

Lemma 4.9. Suppose that the market is arbitrage free and verifies the $P$-property and the ${ }^{*}$-property. Take $K \in \mathcal{P}_{F}(I)$ and a countable collection $\left(B_{n}\right)_{n \in \mathbb{N}} \subset \mathcal{B}_{K}$ such that $\mu_{K}\left(B_{n}\right) \neq 0$ for every $n \in \mathbb{N}$. Then the projective system $\left(\lambda_{J}\right)_{J \in \mathcal{P}_{F}(I)}$ of Lemma 4.8 may be constructed in such a way that $\lambda_{K}\left(B_{n}\right) \neq 0$ for every $n \in \mathbb{N}$.

${ }^{6}$ It may be worthwhile to remark that Assumption 1 is not necessarily crucial when proving the lemma above. Indeed, if it fails then the role of $\mathcal{C}\left(C_{J}\right)$ and $\mathcal{C}^{*}\left(C_{J}\right)$ may be substituted by $L^{\infty}\left(\mathbb{R}^{J}, \mathcal{B}_{J}, \mu_{J}\right)$ and its dual $L_{\infty}^{*}\left(\mathbb{R}^{J}, \mathcal{B}_{J}, \mu_{J}\right)$, space of finitely additive real valued measures on $\mathcal{B}_{J}$ with finite variation and vanishing on every $\mu_{J}-$ null set. 
Proof. We will use the same notation as in the proof of Lemma 4.8. Bearing in mind 4.8.3 consider the projective system $\left(\lambda_{J}^{n}\right)_{J \in \mathcal{P}_{F}(I)}$ such that

$$
\lambda_{K}^{n}\left(B_{n}\right)>0
$$

for every $n \in \mathbb{N}$. Let $\left(\epsilon_{n}\right)_{n \in \mathbb{N}}$. be a decreasing sequence of positive real numbers such that

$$
\sum_{n=0}^{\infty} \epsilon_{n}=1 .
$$

Take finally

$$
\lambda_{J}=\sum_{n=0}^{\infty} \epsilon_{n} \lambda_{J}^{n}
$$

for every $J \in \mathcal{P}_{F}(I)$. The convergence in both the norm topology and the weak $k^{*}$-topology of $\mathcal{C}^{*}\left(C_{J}\right)$ is guaranteed by the Weierstrass criterion. Thus, it is easy to see that $\left(\lambda_{J}\right)_{J \in \mathcal{P}_{F}(I)}$ is a projective system that verifies 4.8 .1 and 4.8 .2 and such that $\lambda_{J}$ is $\mu_{J}$-continuous for every $J \in \mathcal{P}_{F}(I)$. Moreover, $\lambda_{K}\left(B_{n}\right) \neq 0$ trivially follows from (4.11) and $\lambda_{K} \geq \epsilon_{n} \lambda_{K}^{n}$ for every $n \in \mathbb{N}$.

Remark 4.10. Consider an arbitrary positive measure space $\left(W, \sum, \theta\right)$. It is worth to recall the Saks Lemma (see [22]) guaranteeing that for every $\varepsilon>0$ there exists a disjoint partition $W_{1}, W_{2}, \ldots, W_{s}, W_{s+1}, \ldots W_{r}$ of $W$ such that $W_{1}, W_{2}, \ldots, W_{s}$ are $\theta$-atoms and $\theta\left(W_{i}\right) \leq \varepsilon, i=s+1, \ldots, r$. One can apply again this lemma on each $W_{i}, i=s+1, \ldots, r$, and for $\varepsilon / 2$. By induction, it is easy to prove the existence of a disjoint sequence $\left(W_{n}\right)_{n \in \mathbb{N}}$ such that the restriction of $\theta$ to $W_{0}$ is non-atomic and $W_{n}$ is an atom for $n=1,2, \ldots$.

Lemma 4.11. Suppose that the market is arbitrage-free and satisfies the $P$-property and the ${ }^{*}$-property. Take $K \in \mathcal{P}_{F}(I)$. Then the projective system $\left(\lambda_{J}\right)_{J \in \mathcal{P}_{F}(I)}$ of Lemma 4.8 may be constructed in such a way that $\lambda_{K}\left(B_{K}\right) \neq 0$ for every Borel set $B_{K} \subset \mathbb{R}^{K}$ such that $\mu_{K}\left(B_{K}\right)>0$ and $B_{K}$ is an open set or a $\mu_{K}$-atom.

Proof. According to Remark 4.10, consider a partition $\left(W_{n}\right)_{n \in \mathbb{N}}$ of $\mathbb{R}^{K}$ such that $\mu_{K}$ is non-atomic on $W_{0}$ and $\left(W_{n}\right)_{n=1}^{\infty}$ are $\mu_{K}$-atoms. Besides, take a countable basis $\left(G_{n}\right)_{n=1}^{\infty}$ of the usual topology of $\mathbb{R}^{K}$. Then Lemma 4.9 ensures that the projective system may be constructed in such a way that $\lambda_{K}$ does not vanish on those elements of

$$
\left(W_{n}\right)_{n=1}^{\infty} \cup\left(G_{n}\right)_{n=1}^{\infty}
$$

with measure $\mu_{K}$ positive. Thus the lemma trivially follows.

Proof. of Theorem 4.7. Suppose that there exists the projectively equivalent riskneutral measure $\nu_{I}$ and consider the model $\left(S_{i}\right)_{i \in I} \cup\left(S_{\varphi}\right)$ where the price $p_{\varphi}$ of $S_{\varphi}$ is given by (4.2). Then the model $\left(S_{j}\right)_{j \in H} \cup\left(S_{\varphi}\right)$ is trivially arbitrage-free for every $H \in \mathcal{P}_{F}(I)$ with $H \supset J$. Now, one can prove that $\left(S_{j}\right)_{j \in I} \cup\left(S_{\varphi}\right)$ is arbitrage free by proceeding as in the proof of Proposition 3.1. 
In order to prove 4.7 .2 take $\left(J_{n}\right)_{n=1}^{\infty}$ for $n=1,2, \ldots$. According to the latter lemma, for every natural $m=1,2, \ldots$ take the projective system

$$
\left(\lambda_{J_{n}}^{m}\right)_{n=1}^{\infty}
$$

such that $\lambda_{J_{m}}^{m}\left(B_{m}\right)>0$ if $\mu_{J_{m}}\left(B_{m}\right)>0$ and $B_{m}$ is open or $\mu_{J_{m}}$-atom. Take finally a sequence $\left(\epsilon_{m}\right)_{m=1}^{\infty} \subset \mathbb{R}$ as in the proof of Lemma 4.9 , i.e., positive, decreasing and satisfying $\sum_{m=1}^{\infty} \epsilon_{m}=1$. Set

$$
\nu_{J_{n}}=\sum_{m=1}^{\infty} \epsilon_{m} \lambda_{J_{n}}^{m}
$$

$n=1,2, \ldots$ Once again, as in the proof of Lemma 4.9, the Weierstrass criterion guarantees the convergence in the norm topology. It only remains to show the implication

$$
\mu_{J_{m}}\left(B_{m}\right)>0 \Longrightarrow \nu_{J_{m}}\left(B_{m}\right)>0 .
$$

Since we are dealing with Radon measures one can assume that $B_{m}$ is compact and included in $C_{J_{m}}$. If $B_{m}^{o}$ denotes the interior of $B_{m}$ and $\mu_{J_{m}}\left(B_{m}^{o}\right)>0$ then $\nu_{J_{m}}\left(B_{m}^{o}\right)>0$. Otherwise we have that $B_{m} \backslash B_{m}^{o}$ is a compact set with void interior and positive $\mu_{J_{m}}$-measure. The ${ }^{* *}$-property implies that $B_{m} \backslash B_{m}^{o}$ contains a $\mu_{J_{m}}$-atom with positive $\mu_{J_{m}}$-measure. Whence $\nu_{J_{m}}\left(B_{m} \backslash B_{m}^{o}\right)>0$.

Remark 4.12. Theorem 4.1 points out that completeness is a sufficient condition to guarantee the existence of projectively equivalent risk-neutral measures and that new securities can be priced in arbitrage free models. Nevertheless it is worthwhile to illustrate that completeness is far from necessary. Indeed, many alternatives may be given in order to ensure the fulfillment of the implication 3.3.4) $\Rightarrow 3.3 .3$ ).

A significant alternative arises if one applies those findings of Balbás et al. [4]. So we can consider a discrete-time dynamic price process

$$
S(\omega, t)=\left(S_{0}(\omega, t), S_{1}(\omega, t), \ldots, S_{m}(\omega, t)\right): \Omega \times\left\{0<t_{1}<t_{2}<\ldots\right\} \longrightarrow \mathbb{R}^{m+1}
$$

with a finite number $m+1 \in \mathbb{N}$ of assets and an infinite number $\left\{0<t_{1}<t_{2}<\ldots\right\}$ of trading dates. As usual the price process must be adapted to the arrival of new information. Under this framework the arbitrage absence does not imply the existence of martingale measures, as established in Back and Pliska [2]. But the study of Balbás et al. [4] proves the existence of projectively equivalent martingale measures, regardless the completeness of the model. Thus if we consider the oneperiod model with infinite securities such that

for every $(a, b) \in I$ and

$$
\begin{gathered}
I=\{0,1, \ldots, m\} \times\left\{t_{1}<t_{2}<\ldots\right\}, \\
p_{(a, b)}=S_{a}(\omega, 0)
\end{gathered}
$$

$$
f_{(a, b)}(\omega)=S_{a}(\omega, b)
$$

for every $(a, b) \in I$ and almost every $\omega \in \Omega$, then the equivalence between 3.3.3) and 3.3.4) will hold for incomplete markets too. This kind of models may be called "finitely generated" and, as already said in the introduction, our Example 1 is a 
particular case that arises from the counter-example of Back and Pliska [2] (for which $m=1$ ). In some sense, the existence of Example 2 illustrates that one-period models with infinite and countable cardinal of securities are "more general" than dynamic-discrete-time models with a finite collection of securities.

\section{Conclusions}

Representation Theorems have shown to be crucial in Asset Pricing and Mathematical Finance. Regarding markets with infinite number of securities the characterization of the absence of arbitrage by the existence of equivalent martingale measures fails in general.

This paper draws on the projective system approach in order to establish the equivalence between the absence of arbitrage and the existence of projectively equivalent martingale measures, which provides pricing rules allowing for the valuation of new assets. The analysis seems to be quite general since there are no conditions on the set of assets or on the properties of future prices.

The equivalence holds for many significant cases like complete or finitely generated markets. Moreover, since finitely generated markets can in some sense extend many dynamic pricing models, it seems that the analysis of markets with infinitely many securities may deserve important attention in Mathematical Finance.

Projectively equivalent pricing rules have been also found for more complex markets. Under some regularity properties, only the possibility of pricing new securities is necessary and sufficient.

The projective system approach allows us to enlarge the set of states of nature and to identify this set and the set of real prices. Thus a complete equivalence between the initial probability measure and the martingale measure does not hold in general. However, the existence of densities between "real" probabilities and "risk-neutral" ones is guaranteed by introducing the concept of "projective equivalence", which implies that both the martingale measure and the initial probability measure generate equivalent projections.

\section{References}

[1] C.D. Aliprantis, M. Florenzano, V.F. Martins-da-Rocha, R. Tourky, Equilibrium analysis in financial markets with countable many securities, J. Math. Econom. 40 (2004), 683-699.

[2] K. Back, S.R. Pliska, On the fundamental theorem of asset pricing with an infinite state space, J. Math. Econom. 20 (1991), 1-18.

[3] A. Balbás, R. Balbás, S. Mayoral, Risk-neutral valuation with infinitely many trading dates, Math. Comput. Modeling (2007), to appear.

[4] A. Balbás, M. Mirás, M.J. Muñoz-Bouzo, Projective system approach to the martingale characterization of the absence of arbitrage, J. Math. Econom. 37 (2002), 311-323. 
[5] G. Chamberlain, M. Rothschild, Arbitrage, factor structure, and mean-variance analysis on large assets, Econometrica 51 (1983), 1281-1304.

[6] S.A. Clark, The valuation problem in arbitrage price theory, J. Math. Econom. 22 (1993), 463-478.

[7] R.C. Dalang, A. Morton, W. Willinger, Equivalent martingale measures and noarbitrage in stochastic securities market models, Stoch. Stoch. Rep. 29 (1990), 185201.

[8] F. Delbaen, W. Schachermayer, The fundamental theorem of asset pricing for unbounded stochastic processes, Math. Ann. 312 (1998), 215-250.

[9] D. Duffie, D. Filipović, W. Schachermayer, Affine processes and applications in finance, Ann. Appl. Probab. 13 (2003), 984-1053.

[10] M. Harrison, D.M. Kreps, Martingale and arbitrage in multiperiod security markets, J. Econom. Theory 20 (1979), 381-408.

[11] J. Jacod, A. Shiryaev, Local martingales and the fundamental asset pricing theorems in the discrete-time case. Finance Stoch. 2 (1998), 259-273.

[12] E. Jouini, H. Kallal, Martingales and arbitrage in securities markets with transaction costs, J. Econom. Theory 66 (1995), 178-197.

[13] E. Jouini, H. Kallal, Efficient trading strategies in presence of market frictions, Rev. Financial Stud. 14 (2001), 343-369.

[14] Y. Kabanov, D. Kramkov, Asymptotic arbitrage in large financial markets, Finance Stoch. 2 (1998), 143-172.

[15] Y. Kabanov, M. Rasonyi, C. Stricker, No arbitrage criteria for financial markets with efficient friction, Finance Stoch. 6 (2002), 371-382.

[16] Y. Kabanov, C. Stricker, The Harrison-Pliska arbitrage pricing theorem under transaction costs, J. Math. Econom. 35 (2001), 185-196.

[17] I. Klein, A fundamental theorem of asset pricing for large financial markets, Math. Finance 10 (2000), 443-458.

[18] P.E. Kopp, Martingales and stochastic integrals, Cambridge University Press, Cambridge, 1984.

[19] D. Kreps, Arbitrage and equilibrium in economies with infinitely many commodities, J. Math. Econom. 8 (1981), 15-35.

[20] K. Musial, Projective limits of perfect measure spaces, Fund. Math. 40 (1980), 163189.

[21] R.T. Rockafellar, S. Uryasev, M. Zabarankin, Optimality conditions in portfolio analysis with general deviations measures, Math. Program., Ser. B 108 (2006), 515-540.

[22] S. Saks, Addition to the note on some functionals, Trans. Amer. Math. Soc. 35 (1933), 965-970.

[23] W. Schachermayer, A Hilbert space proof of the fundamental theorem of asset pricing in finite discrete time, Insurance Math. Econom. 11 (1992), 249-257.

[24] W. Schachermayer, The fundamental theorem of asset pricing under proportional transaction costs in finite discrete time, Math. Finance 14 (2004), 19-48.

[25] L. Schwartz, Radon measures on arbitrary topological spaces and cylindrical measures, Oxford University Press, London, 1973. 


\section{Acknowledgment}

The authors thank Professor María José Muñoz-Bouzo (Department of Fundamental Mathematics, Spanish Open University) for her useful comments and assistance.

Alejandro Balbás and Anna Downarowicz

University Carlos III of Madrid

C/ Madrid, 126

28903 Getafe (Madrid)

Spain

e-mail: alejandro.balbas@uc3m.es

anna. downarowicz@uc3m.es 